\title{
Heartbeat: afterload is high (not low) in chronic mitral regurgitation!
}

Physicians often use a pathophysiologic framework to understand the clinical presentation of cardiac disease and to justify a specific therapeutic intervention. However, our understanding of pathophysiology typically is based on low quality evidence or 'common sense' thinking, both of which often turn out to be wrong when rigorous studies are performed. For example, many clinicians erroneously believe that left ventricular (LV) afterload in low in patients with mitral regurgitation (MR) due to a low impedance leak into the left atrium. In this issue of Heart, Gaasch and colleagues ${ }^{1}$ provide a mathematical model, with validation based on detailed hemodynamics measurements in a small group of patients, that convincingly demonstrates that retrograde impedance (backflow into the left atrium) exceeds forward impedance (forward flow into the aorta) in patients with severe MR and a normal LV ejection fraction, until regurgitant fraction exceeds 56\%. (figure 1) Despite a high retrograde impedance, total impedance (retrograde plus forward) remains low in patients with chronic MR only because LV enlargement allows a normal LV stress-shortening relation.

In an editorial, Martinez-Legazpi, Yotti and Bermejo $^{2}$ point out the limitations of using the concept of impedance to characterise valve lesions (figure 2). They conclude: 'the concept of chronic MR as a disease of pure high-preload and low-afterload is misleading. Afterload is actually normal or above normal in chronic phases of the disease. In addition to increased preload, the abolishment of isovolumic phases is the source of singular biomechanical consequences on the LV. These issues need to be taken into account for a deeper understanding of the ventricular compensatory mechanisms and for making the right clinical decisions in a time of rapidly evolving therapeutic opportunities'.

Dr Blase Carabello provides an even more provocative commentary ${ }^{3}$ entitled: 'A tragedy of modern cardiology: using ejection fraction to gauge LV function

Correspondence to Professor Catherine M Otto, Division of Cardiology, University of Washington, Seattle 98195, Washington, USA; cmotto@uw.edu in MR'. He puts the current study in context with his comment: 'The goal in valve disease is to time surgery before LV dysfunction, and its consequences impact outcome. Currently, we are using a 50-year-old load-dependent tool, ejection fraction, to gauge function in the lesion, with the most confusing and least predictable changes in load'. Further, he challenges us to 'develop biological tools that can peer into the workings of the myocardium to understand and predict when LV contractility is beginning to fall and when LV dysfunction will affect prognosis, tools that can and must replace our ancient, rusty, dull tool, ejection fraction'.

The increased risk of cardiovascular disease in smokers is well established but data is sparse on relative risks in different age groups. With the goal of accounting for population smoking trends, Lloyd and colleagues ${ }^{4}$ examined incidence rates and rate ratios for risk of ST elevation MI by age group in South Yorkshire

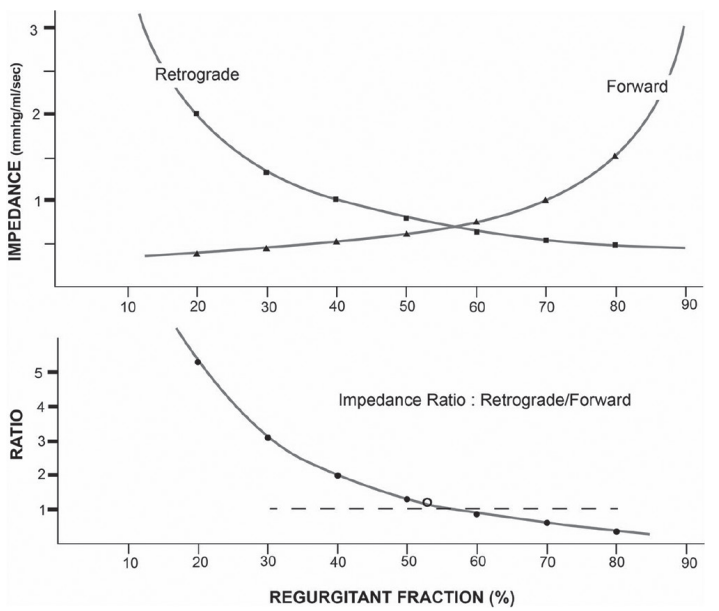

Figure 1 Impedance to retrograde and forward flow in chronic mitralregurgitation. The model is based on an end-diastolic volume of $180 \mathrm{~mL}$, an ejection fraction of $60 \%$ and a left ventricular mean systolic pressure of $110 \mathrm{~mm} \mathrm{Hg}$. The durations of retrograde and forward flow were taken as 400 and $300 \mathrm{~ms}$, respectively. In the upper panel, the impedance to retrograde flow (closed squares) is plotted against regurgitant fraction; the coordinates were calculated over a wide range of regurgitant fractions (at intervals of 10\%). Likewise, the impedance to forward flow (closed triangles) is plotted against regurgitant flow over the same range of regurgitant fractions. The impedance to retrograde flow is greater than forward flow over a wide range of regurgitant fractions up to $57 \%$. Only when the regurgitant fraction exceeds $57 \%$ is the impedance to retrograde flow less than to forward flow. In the lower panel, the ratio of retrograde to forward impedance (closed circles) is plotted against regurgitant fraction. The model indicates that a ratio exceeding one (broken line) reflects a higher impedance to retrograde flow than to forward flow. The average ratio $(1.22 \pm 0.19)$ of the patient group with a regurgitant fraction of $53 \pm 4 \%$ (open circle) is superimposed on the model. 


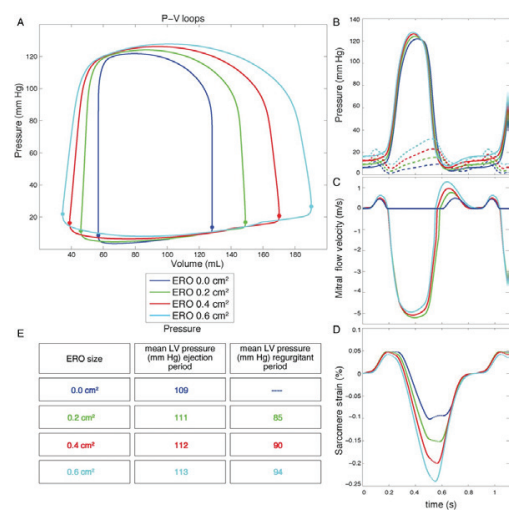

Figure 2 Haemodynamic changes induced by progressive effective regurgitant orifices (ERO) of mitral regurgitation (MR). (A) Left ventricular (LV) pressure-volume loops. The disappearance of isovolumic phases is visualised as the progressive angulation of the vertical segments of the loop of $E R O=0.0 \mathrm{~cm} 2$. Bullets depict the onset and end of the regurgitant period. (B) LV (solid lines) and atrial (dashed lines) pressure evolution with time. (C) Transmitral flow velocity, showing progressive shortening of the regurgitant time with increasing degrees of MR. (D) Sarcomere strain; dashed lines account for isovolumic chamber periods. When $E R O=0 \mathrm{~cm} 2$, isometric contraction takes place with a flat strain and relaxation takes place during sarcomere lengthening (strain is increasing). MR causes both phases to take place while the sarcomere is shortening (strain is decreasing). (E) Values of mean LV pressure during the ejection and regurgitation phases. Simulations were performed using CircLab software, 12 at $75 \mathrm{bpm}$, and set to autoregulate for a mean systolic arterial pressure of $92 \mathrm{~mm}$ $\mathrm{Hg}$ in all cases.

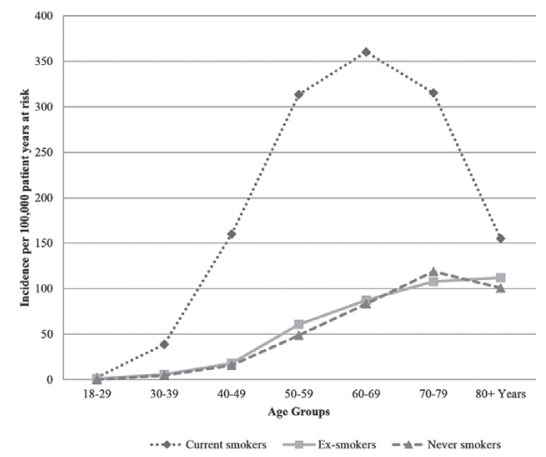

Figure 3 Incidence of acute ST-segment elevation myocardial infarction(STEMI) by smoking status in South Yorkshire, calculated using patient time from the Office of National Statistics Integrated Household Survey (ONSIHS) and a cohort of patients presenting with STEMI. Current smokers had a peak incidence of acute STEMI at age 60-69 years, whereas for ex- and never smokers, the peak incidence was 10 years later at $70-79$ years.
The increasing role of advanced imaging modalities for elucidating mechanisms of disease is illustrated by the study of Jenkins and colleagues ${ }^{6}$ which used positron emission tomography (PET), CT imaging and cardiac magnetic resonance imaging (CMR) to study cardiac repair and recovery after recent MI (figure 4). The ability to visualise changes in molecular activity at the tissue level in living patients

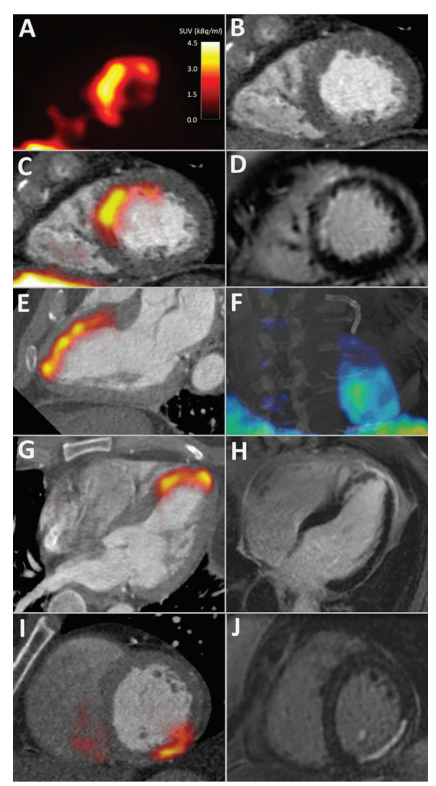

Figure 4 18F-Fluciclatide uptake in acute myocardial infarction (MI) is shown. 18F-Fluciclatide uptake in three patients with recent subendocardial Ml is shown. Patient 1, 13 days after anterior MI, displaying a shortaxis positron emission tomography (PET) image of the left ventricle with crescentic 18F-fluciclatide uptake (A) that correlates with the interventricular septum and anterior wall on CT angiography (B). The fused PET/ CT-angiography image (C) shows this uptake to correspond exactly with the region of late gadolinium enhancement (LGE) on cardiac magnetic resonance (CMR) (D). Further delineation of myocardial uptake on PET/CT is clearer in the two-chamber view $(\mathrm{E})$ and on a fused CT/three-dimensional-Patlak image, which shows this uptake to follow a watershed-pattern emerging from the coronary stents present in the left anterior descending coronary artery (F) (see online supplementary video 1). (G) and (H) Patient 2, 8 days following anterior $\mathrm{MI}$, displaying focal uptake of 18Ffluciclatide in the anterior wall and apex in the three-chamber view on PET/CT (G) which corresponds to the region of infarction on LGE CMR imaging (H). (I) and (J) Patient 3, showing focal uptake of $18 \mathrm{~F}$-fluciclatide in the inferior wall 19 days following MI on PET/CT (I) that again corresponds to the infarction on CMR LGE imaging (J). promises to revolutionise treatment of cardiac disease over the next few decades.

The Education in Heart article ${ }^{7}$ in this issue focuses on transoesophageal echocardiography, including indications, risks, pitfalls, and diagnostic value of this imaging modality.

The Image Challenge ${ }^{8}$ in this issue requires correct interpretation of an ECG in a young man with exercise induced syncope. These board review style multiple choice questions are a great way to prepare for exams. They are also a fun and easy way to update your knowledge of clinical cardiology with a short discussion related to a clinical image. You can find all the Image Challenges questions on our new website by using the Advanced Search function and searching for the phrase 'Image Challenge' in the 'Full Text or Abstract or Title' box.

\section{Competing interests None declared.}

Provenance and peer review Commissioned; internally peer reviewed.

(C) Article author(s) (or their employer(s) unless otherwise stated in the text of the article) 2017. All rights reserved. No commercial use is permitted unless otherwise expressly granted.

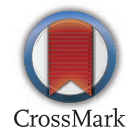

To cite Otto CM. Heart 2017;103:565-566.

Published Online First 9 March 2017

Heart 2017;103:565-566.

doi:10.1136/heartjnl-2017-311510

\section{REFERENCES}

1 Gaasch WH, Shah SP, Labib SB, et al. Impedance to retrograde and forward flow in chronic mitral regurgitation and the physiology of a double outlet ventricle. Heart 2017; 103:581-5.

2 Martinez-Legazpi P, Yotti R, Bermejo J. How heavy is the load? the ventricular mechanics of mitral regurgitation revisited in the era of percutaneous therapies. Heart 2017;103:567-9.

3 Carabello BA, Carabello BA. A tragedy of modern cardiology: using ejection fraction to gauge left ventricular function in mitral regurgitation. Heart 2017; 103:570-1.

4 Lloyd A, Steele L, Fotheringham J, et al. Pronounced increase in risk of acute ST-segment elevation myocardial infarction in younger smokers. Heart 2017;103:586-91.

5 Arbel Y. When will we learn that smoking is bad? Heart 2017;103:572

6 Jenkins WS, Vesey AT, Stirrat C, et al. Cardiac $\alpha v \beta 3$ integrin expression following acute myocardial infarction in humans. Heart 2017;103:607-15

7 Wamil M, Newton JD, Rana BS, et al. Transoesophageal echocardiography: what the general cardiologist needs to know. Heart 2017; 103:629-40.

8 Yeo C, Tan VH, Wong KCK. Exercise-induced Syncope in a 22-year-old man. Heart 2017;103:591. 